\title{
Effect of Monopile Foundation Modeling on the Structural Response of a 5-MW Offshore Wind Turbine Tower
}

\author{
Sungmoon Jung ${ }^{\text {a }}$, Sung-Ryul Kim ${ }^{\text {b* }}$, Atul Patil ${ }^{c}$, Le Chi Hung ${ }^{d}$ \\ ${ }^{a}$ Associate Professor, Department of Civil and Environmental Engineering, Florida A\&M University - Florida \\ State University College of Engineering, Tallahassee, FL, USA. Email: sjung@eng.fsu.edu \\ ${ }^{\mathrm{b}}$ Associate Professor, Department of Civil Engineering, Dong-A University, Busan, Korea. Email: \\ sungryul@dau.ac.kr (Corresponding author), Phone:+82-51-200-7622 \\ ${ }^{\mathrm{c}}$ Graduate Research Assistant, Department of Civil and Environmental Engineering, Florida A\&M University \\ - Florida State University College of Engineering, Tallahassee, FL, USA \\ ${ }^{\mathrm{d}}$ Manager, Civil, Structure \& Arch. Engineering Team,Global Engineering Technology, Seoul, Korea
}

\begin{abstract}
Offshore wind turbine towers experience large base moments because of wind and wave loading. The flexibility of the foundation should be considered when analyzing the structural response of towers. Previous studies showed that conventional p-y curves are not suitable in designing the foundation. More advanced methods, such as the finite element method, are necessary to model the offshore wind turbine foundation. In addition, these studies focused on the analysis of the foundation itself, so the effect on the structural response of the tower merits further research. The present study aimed to compare different foundation modeling approaches, focusing on their effects on the structural response of the wind turbine tower. We integrated wind turbine aerodynamic simulation with different models of the foundation. We confirmed that ignoring the flexibility of the foundation caused significant error in wind turbine tower behaviour. Between the p-y curve-based model and finite element-based model, the change in maximum moment was insignificant, but the maximum tilt angle increased over $14 \%$ in the finite element model. Therefore, the finite element approach is recommended to obtain a conservative design when large tilt angles may cause serviceability issues.
\end{abstract}

Keywords: wind turbine tower, monopile, offshore, foundation, aerodynamics, finite element analysis

(C) 2015. This manuscript version is made available under the Elsevier user license

http://www.elsevier.com/open-access/userlicense/1.0/ 


\section{Introduction}

Offshore wind turbine structures are distinguished from other offshore structures, such as oil and gas platforms. The tower is slender with large lateral forces at the top, thereby experiencing large moments at the tower base.Consequently, they pose new challenges in the analysis, design, and construction of the foundation. The foundation should not only support vertical loading but also prevent failure caused by the large moment. Foundation types for offshore wind turbines include monopiles, gravity bases, space-frames, and floating structures. The monopile foundation is the most commonly used type for shallow waters (less than $30 \mathrm{~m}$ in depth), which is the focus of this paper.

One of the earliest studies on the foundation modeling of offshore wind turbines was by Zaaijer (2002). The author compared the distributed spring model, cantilever with the effective fixity length, stiffness matrix-based approach, and uncoupled spring model. The stiffness matrix at the mudline was found to be a good approximation for modeling foundation flexibility. Van der Tempel (2006) discussed a similar approach for foundation modeling. Recent studies investigated the foundation effect more thoroughly while considering wind turbine aerodynamics (Jonkman et al., 2007; Bush and Manuel, 2009); however, these studies relied on conventional p-y curve approach in modeling the effect of soil. The p-y curve approach is commonly used for other offshore structures, such as oil platforms(API, 2007).A recent report highlighted the limitation of using conventional models for offshore wind turbine foundations (TRB 2011). This report stated that "monopile substructures for wind turbines exceed the diameters and experience base of the oil and gas industry" and "extrapolating current practice to the larger sizes can introduce unintended effects"(TRB, 2011, p.57). Previous studies also pointed out that conventional p-y curvesmay not be suitable for the design of offshore wind turbine foundation, and the finite element method (FEM) has been used as a more 
accurate alternative. These studies include the works by Lesny and Wiemann (2006), Lesny et al. (2007), Sørensen et al. (2009), Hearn(2009),Hearn and Edgers (2010), and Achmus and Abdel-Rahman (2012).

The present study aimed to compare p-y curve-based foundation modeling andFEM-based foundation modeling, while considering wind turbine aerodynamics. Although new studies on FEM-based modeling exist, our understanding of the effect of foundation modeling on wind turbine tower behaviour is quite limited. By integrating wind turbine aerodynamic simulation and FEM-based foundation modeling, we aimed to quantify the effect on the response of the wind turbine tower. Structural responses of the tower will be compared while changing foundation modeling. Fixed boundary, p-y curve-based spring model, and FEM-based model were also compared in this paper.

\section{Description of the wind turbine and foundation}

We used the 5 MW reference wind turbine from the National Renewable Energy Laboratory (NREL) in the analysis (Jonkman et al., 2009). The NREL developed the reference turbine to aid concept studies and research activities on offshore wind energy.

Fig. 1 shows a schematic of the reference wind turbine. It has three blades with variable speed and pitch control. The cut-in, rated, and cut-out wind speeds are 3, 11.4, and 25 $\mathrm{m} / \mathrm{s}$, respectively. The rotor diameter is $126 \mathrm{~m}$. The tower height is $87.6 \mathrm{~m}$, and the water depth is $20 \mathrm{~m}$. The masses of the rotor, nacelle, and tower are 110,240 , and 347.5 ton, respectively. The tower hasa base diameter of $6 \mathrm{~m}$ (wall thickness $=0.027 \mathrm{~m})$ and top diameter of $3.87 \mathrm{~m}$ (wall thickness $=0.019 \mathrm{~m})$. Further details on the wind turbine can be found in Jonkman et al. (2009).

The support structures used for offshore wind turbines include monopiles, gravity bases, space-frames, and floating structures. This study investigated the monopile 
foundation, which is the most commonly used type for shallow waters (less than $30 \mathrm{~m}$ in depth). The monopiles can be installed into sandy and clayey soils by driving or vibrating. These soils can be found widely at different offshore sites, such as the North Sea or Baltic Sea in Europe for sandy soil, and the Gulf of Mexico or Western Australia for clayey soils. In this study, two representative soil profiles were assumed, which were adapted from the works of Passon(2006) (for sandy soil) and Chen et al.(2009) (for clayey/sandy soil), as shown in Fig.2. The sandy soil profile was named "stiff soil", whereas the clayey and sandy soil profile was named "soft soil". In the figure, the parameters $\gamma^{\prime}, E^{\prime}\left(E_{u}\right), v^{\prime}\left(v_{u}\right), \phi^{\prime}, \Psi^{\prime}, c^{\prime}$ and $s_{\mathrm{u}}$ present the effective unit weight, Young's modulus, Poisson's ratio, effective friction angle, dilation angle, cohesion and undrained shear strength of soils, respectively. For p-y curve analyses, the soil modulus $k$ in sand was selected based on the $\phi$ ' values following recommendations of the API sand models (API, 2007; Isenhower and Wang, 2012), and the soil strain parameter $\varepsilon_{50}$ in clay was determined based on the undrained shear strength of clay(Matlock, 1970; Isenhower and Wang, 2012).

The steel pile with adiameter of $6 \mathrm{~m}$ was assumed to be driven up to 36 and $40 \mathrm{~m}$ into the stiff soil and soft soil, respectively. The wall thickness of the piles was set to 6 $\mathrm{cm}$. The piles had a Young's modulus $\left(E_{\mathrm{s}}\right)$ of $210,000 \mathrm{MPa}$ with a yield stress $\left(\sigma_{\mathrm{y}}\right)$ of $205 \mathrm{MPa}$.

\section{Modeling of the foundation behaviour}

\subsection{Coupled spring model}

The monopile and soil influence the structural response of the wind turbine. To model the effect of the foundation, we employed the coupled spring model shown 
inFig.3 (Bush and Manuel, 2009). Other researchers have shown that this approach accurately models the effects of soil in wind turbine simulation(Jonkman et al, 2007).

The first step in the coupled spring approach is to define the flexibility matrix as follows:

$$
\left\{\begin{array}{l}
u_{h} \\
u_{r}
\end{array}\right\}=\left[\begin{array}{ll}
S_{h h} & S_{h r} \\
S_{r h} & S_{r r}
\end{array}\right]\left\{\begin{array}{l}
F_{h} \\
F_{r}
\end{array}\right\}
$$

in which the two-by-two matrix is the flexibility matrix, $u_{h}$ is the lateral displacement,

$u_{r}$ is the rotation, $F_{h}$ is the lateral force, and $F_{r}$ is the moment. The displacement and force are from the top of the pile (the mudline). The first column of the flexibility matrix $\left(S_{h h}, S_{r h}\right)$ can be obtained by applying the lateral force only (while $\left.F_{r}=0\right)$ and recording the displacement and rotation. Similarly, the second column of the flexibility matrix can be obtained by applying the moment only and recording the displacement and the rotation. Once the flexibility matrix is obtained, the following stiffness matrix can be determined by inverting it:

$$
\left\{\begin{array}{l}
F_{h} \\
F_{r}
\end{array}\right\}=\left[\begin{array}{ll}
K_{h h} & K_{h r} \\
K_{r h} & K_{r r}
\end{array}\right]\left\{\begin{array}{l}
u_{h} \\
u_{r}
\end{array}\right\}
$$

in which the two-by-two matrix is the stiffness matrix. To use the coupled spring model, we need to obtain force-displacement curves, which will be explained in the next section.

\subsection{Soil modelsand validation of finite elementmodeling}

Three different soil modeling approaches, namely, the fixed boundary model, p-y curve-based spring model, and FEM-based spring model were compared in this study. The fixed boundary model ignores the flexibility of the soil. The bottom of the wind turbine is modeled as completely fixed. This approach is inaccurate, it will be analysed and comparedwith other approaches. 
The p-y curve is a commonly used method to analyze the horizontal loaddisplacement behaviour of piles. The springs in the p-y-curve method represent soil behaviour. Theoretically, the p-y curve method is based on the theory of subgrade reaction, representing the soil horizontal resistance per unit length (p) of a pile when the pile is translated laterally by a displacement (y) into the soil. The p-y method was implemented through the LPILE V6.31 program (Isenhower and Wang, 2012 ) with the API sand model (O’Neil and Murchison, 1983) and soft clay model (Matlock, 1970). The Clay 3 layer in Fig. $2 b$ is considered as a stiff clay layer, so this layer was simulated by the stiff clay model (Reese et al., 1970).

For the FEM-based spring model, the foundation was modeled using 3D solid elements. Fig. 4 shows a typical finite element (FE) mesh used in the analysis. Loaddisplacement curves were obtained from the FEM simulations, which were then converted to equivalent coupled springs. Small-strain FE analysis was conductedusing commercial Abaqus software (Simulia, 2010). The continuum element type C3D8R was adapted to model the soil and pile. The constitutive models obeyed the Mohr-Coulomb and Tresca failure criteria to simulate the behaviour of sand and clay ( $\phi=0$ condition), respectively. The interface between the pile and soil was simulated by the Coulomb friction law, and detachment between the pile and soil was allowed. The frictional coefficient at the interface was simply taken as $\tan \left(2 / 3 \phi^{\prime}\right)$ for sand, and the interface shear strength between the pile and clay was assigned similar to the undrained shear strength of clay.

The accuracy of FE modeling adopted in this study was validated by comparing the obtained data with the published experimental test data as shown in Fig. 5. Peng (2006) reported a series of $1-\mathrm{g}$ experimental tests for monopiles subjected to horizontal loads. The piles were installed in sandy soil. A result of the static horizontal loadingtest 
was compared with a result of FE analysis in this study. The properties of sandy soil used for FE analysis were determined from laboratory test results, which can be found in the work of Peng (2006). Kim et al. (2014) reported a series of centrifuge tests for the bucket foundations (tripod bucket and single bucket foundations) subjected to horizontal loads. A static test result of the single bucket foundation was adopted for comparison with FE analysis. The foundation was embedded in silty sand. The soil properties used for FE analysis were determined based on the CPT data presented in Kim et al. (2014).

The Mohr-Coulomb failure criterion was used to simulate the stress-strain behaviour of sandy soils, whereas the monopile and bucket foundation were considered as linear elastic materials in FE analysis. The comparison between FE analyses and the test results showed that the horizontal load-displacement curves obtained from FE analses were in good agreement with those measured from experimental tests. In addition, the accuracy of FE analysis has been extensively verified, and can be found in the studies of Achmus et al. (2013) (for sand), Jeanjean (2009), and Hung and Kim (2014) (for clay).Therefore, the numerical modeling approach adopted in this study was confirmed to be reliable.

\subsection{Comparison between FE modeling and p-y curve approach}

Jeanjean (2009) analyzed the lateral resistance of the monopile in undrained clay using the centrifuge test, FE analysis and p-y curve method. A prototype pile, which had the diameter of $0.9 \mathrm{~m}$, length of $36.5 \mathrm{~m}$ and wall-thickness of $50.8 \mathrm{~mm}$, was modeled. The FE results showed almost identical resistance with the centrifuge model tests, whereas the p-y curve results significantly underestimated the soil resistances from the FE and centrifuge results. In addition, the soft clay model of the p-y curve method significantly underestimated soil resistance. 
Hearn and Edgers (2010) extensively investigated the lateral behavior of a large monopile in dense sand using FE analysis and the p-y curve method. The pile was modeled as a linear elastic material, and the Mohr-Coulomb constitutive model was adopted to simulate the behaviour of dense sand. The pile had a diameter of $5 \mathrm{~m}$ and embedment depth of $26 \mathrm{~m}$. The horizontal load-displacement relationships obtained from the p-y curve method and FE analysis were compared. The results showed that the p-y curve method significantly overestimated the horizontal resistance in comparison with FE analysis. Abdel-Rahman and Achmus (2005) investigated the lateral behaviour of a monopile in sand using FE analysis and the p-y curve method. Similar to the work of Hearn and Edgers (2010), the sand was simulated using the Mohr-Coulomb failure criterion, and the pile was considered as a linear elastic material. They stated that the p-y curve method is not applicable to determine the horizontal displacement or rotation for a large monopile for offshore wind turbine.

Based on the above verifications, the FE modeling might be more accurate than the p-y curve method to simulate the horizontal-displacement behaviour of monopiles with large diameter. The p-y curve method used in this study was developed based on the limited number of test piles with small diameters. In addition, the p-y curve method does not properly simulate the true behaviour of interface between the pile and soil (i.e., detachment and sliding), whereas the FE method can correctly model the stress-strain behaviour of soil, true interface between the pile and soil, and change in the pile geometry in practical design.

Fig. 6shows a comparison of LPILE analysis and FEM analysis for stiff soil. The LPILE response was always stiffer than the FEM response as shown in the figure. Fig.7shows a similar comparison for soft soil. Unlike stiff soil, the LPILE response was stiffer than the FEM response up to a certain point, but the trend reversed afterwards. 
These trends between the FEM and LPILE responses (showed in Figs. 6 and 7) were consistent with previous findings. The p-y curve method ususally overestimates the soil resistance of sand (Abdel-Rahman and Achmus, 2005; Hearn and Edgers, 2010), and underestimates the undrained resistance of clay (Jeanjean, 2009). However, the initial slope of the curve in the $\mathrm{p}-\mathrm{y}$ curve method is dependent on the soil modulus $k$ in sand and soil strain parameter $\varepsilon_{50}$ in clay; these parameters are empirically determined from the recommendation in API (2007), and may not be applicable to other specific field conditions. Analysis results of the wind turbine demonstrated that the maximum shear force and maximum moment were about $2.5 \mathrm{MN}$ and $110 \mathrm{MN}-\mathrm{m}$, respectively. However, the average shear force and moment were much smaller than these values. Therefore, for the force and moment experienced by the wind turbine, the LPILE response was almost always stiffer than the FEM response.

\section{Analysis procedure}

\subsection{Analysis method}

Simulations were conducted using FAST software (Jonkman and Buhl, 2005). FAST stands for fatigue, aerodynamics, structures, and turbulence, and these characteristics are key features of the program. FAST employs a combination of modal and multi-body dynamic formulations. Some parts are modeled as rigid bodies (e.g., nacelle, hub, and gear), whereas other parts are modeled as flexible bodies (e.g., blades and tower) using a linear modal representation that assumes small deflections. The parts

primarily relevant to this study are blades, tower, and foundation. For each blade, two degrees of freedom (DOFs) account for the first and second flap-wise mode, and one DOF accounts for the first edge-wise mode. The tower has two DOFs for the longitudinal modes and two DOFs for the lateral modes. Finally, the foundation has 
three translational DOFs (surge, sway and heave) and three rotational DOFs (roll, pitch and yaw). The directions of the DOFs are shown in Fig.8.

FAST uses numerically simulated time-series of three-component wind velocity, at points in a two-dimensional rectangular grid(Jonkman, 2009). The rectangular grid lies on the plane where the wind turbine blades are located. Spectra and spatial coherence are defined in the frequency domain, and time-series can be generated assuming the stationary process.

The generated time-series of wind loading causes forces on the wind turbine blades. FAST calculates the lift, drag, and pitching moment of the airfoil section using relevant information such as turbine geometry, operating condition, and wind inflow(Moriarty and Hansen, 2005). The program then calculates the forces on the wind turbine blades, which cause blade deflections and aeroelastic interactions. The aerodynamics of the blade is based on blade element momentum theory. Further details of the blade aerodynamic formulations can be found in Moriarty and Hansen (2005)and Manwell et al. (2009).

Let $\mathbf{F}_{i}^{A}$ be the resultant of aerodynamic forces acting on the center of the deflected blade element ( $i^{\text {th }}$ blade). Then, generalized active aerodynamic force for the $k^{\text {th }}$ DOF is expressed as follows:

$$
\left.F_{k}\right|_{\text {Aero }}=\sum_{i=1}^{3} \int_{0}^{R-R_{H}} \mathbf{v}_{i k} \cdot \mathbf{F}_{i}^{A} d r_{i}
$$

where $R$ is the total radius of the rotor, $R_{H}$ is the hub radius, $\mathbf{v}_{i k}$ is the partial velocity at the center of the deflected blade element in the inertial reference frame $\left(i^{\text {th }}\right.$ blade, $k^{\text {th }}$ DOF), and $d r$ is the differential length of the element. Detailed derivation of each term and the definition of the reference frame are highly involved, and further detail can be found in Jonkman (2003). 
Similarly, the generalized forces for elastic forces (which include tower and blade), gravitational forces, generator forces, and damping forces can be computed.

$$
F_{k}=\left.F_{k}\right|_{\text {Aero }}+\left.F_{k}\right|_{\text {Elastic }}+\left.F_{k}\right|_{\text {Grav }}+\left.F_{k}\right|_{\text {Generator }}+\left.F_{k}\right|_{\text {Damp }}
$$

Likewise, the generalized inertia forces of the tower, nacelle, hub, and blades can be computed.

$$
F_{k}^{*}=\left.F_{k}^{*}\right|_{\text {Tower }}+\left.F_{k}^{*}\right|_{\text {Nacelle }}+\left.F_{k}^{*}\right|_{\text {Hub }}+\left.F_{k}^{*}\right|_{\text {Blade }}
$$

These forces involve inertia forces (acceleration vectors and mass) and velocity vectors. FAST uses Kane's method to establish equations of motion (Jonkman, 2003). Further derivations using Eqs. (4) (5) give us the following equation.

$$
\sum_{s=1}^{N} C_{k s} \ddot{q}_{s}+f_{k}\left(\dot{q}_{k}, q_{k}\right)=0
$$

where $N$ is the total DOF, $C_{k s}$ 's are coefficients of the accelerations of each DOF variable, and $f_{k}$ 's contain lower order terms. These equations are solved numerically in the time-domain simulation.

\subsection{Updating of the foundation stiffness}

As explained earlier, we represented the effect of the foundation using the coupled spring model shown in Eqs. (1) and (2). Given that default FAST code assumes the fixed boundary at the bottom of the tower, we customized the code and re-compiled it for this research. The subroutine UserPtfmLd was customized to include the effect of the foundation. 
The coupled spring model uses linearized springs. When the soil exhibits nonlinearity, the stiffness matrixused in FAST simulation needs to be updated iteratively. Fig. 9 explains the steps that we used to update the stiffness matrix. $N=10$ and $t o l=0.05$ were used in the example presented in this paper. The initially assumed stiffness was obtained using the values provided in Bush and Manuel (2009). $\mathbf{u}_{\text {avg }}^{i}$ was computed by taking the average of surge, sway, roll, and pitch responses from the $N$ simulations. All cases studied in this paper converged within three iterations.

FAST uses mode shapes of the tower as one of the input files. The mode shapes should reflect the foundation stiffness. Once the converged stiffness was obtained, the mode shapes for FAST were computed.

Note that this procedure is a simplified way of considering soil stiffness. For more accurate analysis, the difference in loading and unloading behaviour of soil should be modeled for the entire time-history of FAST simulation. Moreover, the number of simulations should be increased for other purposes such as prediction of the long-term load.

\section{Effect of monopile foundation modeling on the structural response of the tower}

\subsection{Time-series analysis and data processing}

The following loading conditions were used for the examples presented in this paper. The mean wind speeds at the rotor height were 12 and $18 \mathrm{~m} / \mathrm{s}$. The speed of 12 $\mathrm{m} / \mathrm{s}$ was selected because it isclose to the rated wind speed and it would cause the greatest structural response of the tower. The speed of $18 \mathrm{~m} / \mathrm{s}$ was selected to study the

behaviour in another operating condition, which was approximately the middle point of the rated speed and the cut-out speed. IEC 61400-1 Kaimal spectrum (IEC, 2005) was used as the turbulence model. Normal turbulence model Class B was used. Wave 
loading was modeled using JONSWAP spectrum and the significant wave height was set as $5 \mathrm{~m}$. FAST considers coupling of wind and wave in the following manner. At each time step, the position, velocity, and acceleration of the substructure are obtained, which include the substructure motion caused by wind loading. The hydrodynamic loads are calculated using the position, velocity, and acceleration of the substructure. Morison's equation is used to compute the hydrodynamic loads.

Wave loading was modeled using JONSWAP spectrum and the significant wave height was set as $5 \mathrm{~m}$. The peak-spectral wave period and water density were set as 12.4 seconds and $1027.0 \mathrm{~kg} / \mathrm{m}^{3}$, respectively. The peak-shape parameter followed IEC 61400 3 Annex B (IEC 2009). Previous studies showed that the structural response of the NREL 5 MW wind turbine is governed by the wind loading rather than the wave loading [ex: see Fig. 1 in Agarwal and Manuel (2009)]. Due to the computationally intensive nature of the study, we selected only one significant wave height, following the median value given in Agarwal and Manuel (2009).

For the fixed boundary model, the foundation was assumed to be completely rigid. The DOFs at the tower base were inactive. The time step of 0.0125 was used in this type of simulation. For all other cases, the effect of soil was modeled using equivalent translational and rotational springs at the tower base for the DOFs of surge, roll, sway, and pitch (see Fig.3). Due to the increased number of DOFs, convergence difficulties were experienced in FAST simulations. The time step size was reduced to 0.00125 for all simulations that considered the flexibility of soil.

We ran 100 time-series analyses for each of the five cases: fixed base, LPILE stiff soil, FEM stiff soil, LPILE soft soil, and FEM soft soil. For all cases except the fixed base case, the foundation stiffness was first calibrated as explained in Section 4.2, and 100 simulations were then run. The random seed was reset each time in wave and wind 
field generation, so the time-series response changed for each simulation. All simulations were conducted for $630 \mathrm{~s}$. The initial $30 \mathrm{~s}$ were excluded from postprocessing following the recommendation of FAST. The initial $30 \mathrm{~s}$ typically show the transient response caused by the wind turbine start-up, and they are not representative of the steady state response. The results from the last $600 \mathrm{~s}$ were used in data processing.Wind turbine design loads are often obtained for the 10-min wind speed [e.g., use of the 10-min wind speed $V_{\text {ref }}$ in IEC (2005)], which is why we used a simulation time of 600 s. Fig. 10 shows sample results for the mean wind speed of 18 $\mathrm{m} / \mathrm{s}$ and the significant wave height of $5 \mathrm{~m}$ for a fixed base case. In order to compare all results effectively, we took average and maximum from each simulation. For example, in the figure shown below, we took average base shear of $349.2 \mathrm{kN}$, maximum base shear of $2210 \mathrm{kN}$, average base moment of $40096 \mathrm{kN}-\mathrm{m}$, and maximum base moment of $88680 \mathrm{kN}-\mathrm{m}$ for further comparisons with other cases.

\subsection{Shear forces and bending moments}

Shear forces and bending moments at the mudline were first compared. Following the notation shown in Fig.8, the shear force is along $x$-axis, or along the direction of the wind and the wave, and the bending moment is about $y$-axis. Tables 1 and 2 summarize the average statistics of the shear force and the moment. The value shown under the column "Average" is the average of 100 ten-minute averages, whereas the value shown under the column "Maximum" is the average of 100 ten-minute maximums.

For the loading conditions and approach used in this paper, the difference between the fixed base model and flexible foundation models was smaller than results from similar research conducted on stiff soil (Bush and Manuel, 2009). The trend for soft soil was similar to that for stiff soil. For the shear force and the moment, the difference between the LPILE approach and FEM approach was also insignificant (at most less 
than $4 \%$ difference for the bending moment), which was comparable with previous findings; thus the selection of soil modeling approach did not significantly change the bending moment (Bush, 2009). The results of $12 \mathrm{~m} / \mathrm{s}$ were higher than those of $18 \mathrm{~m} / \mathrm{s}$ because $12 \mathrm{~m} / \mathrm{s}$ was closer to the rated speed, which was also consistent with previous findings. The speed of $12 \mathrm{~m} / \mathrm{s}$ was close to the rated speed, where the largest shear forces and moment were expected. The tables present average statistics, but the maximum values were substantially higher than the average. Figs.11 and 12 show the exceedance probabilities of the bending moment. The plot shows the probability that a certain magnitude of the bending moment will be equaled or exceeded. Although the observed trend was similar to that reported in previous research, the difference between the fixed base model and other models was small. The current research used different wave loadings, stiffness calculation approaches, and a smaller number of simulations, which possibly contributed to the smaller difference. However, only the LPILE results were compared with previous studies, because these studies did not include FEM modeling.

To summarize the main findings, the fixed base model may provide a nonconservative answer, as clearly shown in Fig.11. Therefore, it is important to model the flexibility of the soil. However, both the LPILE approach and FEM approach provided comparable answers, with less than $4 \%$ difference of bending moment between the two approaches.

\subsection{Tilt at pile head}

Wind turbine foundation has performance requirements that must be met to ensure satisfactory operation of the wind turbine. For offshore wind turbines, the maximum allowable tilt at pile head is $0.5^{\circ}$ (Malhotra, 2011). However, the allowable tilt will become smaller if we consider the tilt error that occurs during the construction. Typical 
construction tolerances range from $0.2^{\circ}$ to $0.25^{\circ}$ (Malhotra, 2011). Therefore, if we subtract the construction error up to $0.25^{\circ}$, the maximum allowable tilt at pile head will be $0.25^{\circ}$. The maximum allowable tilt for ensuring satisfactory operation is also called the serviceability state (DNV, 2011).

Notably, the wind turbine analyzed in this paper was well-designed to meet these design criteria. Our simulation results also confirmed that the performance of the support structure was satisfactory. Given that the pitch angle satisfies the allowable limit, the discussions below focus on the difference between the LPILE approach and FEM approach. Tables 3 and 4 show the average statistics of surge and pitch. The surge was insignificant compared with the $6 \mathrm{~m}$ diameter of the pile, but the data are shown for reference. In the rows labeled with "FEM", the percent change compared with the LPILE approach is also shown within the parentheses. The FEM approach produced pitch angles that were higher than the LPILE approach by over $8 \%$ and $14 \%$ for soft soil and stiff soil, respectively.

The above tables show the average statistics, so the maximum pitch angles for some ten-minute incidents were substantially higher than what was shown above. Figs. 13and 14show the exceedance probability using the maximum values of all 100 simulations. Let us discuss the results of the speed at $12 \mathrm{~m} / \mathrm{s}$, which produced higher pitch angles. For the stiff soil case, the maximum pitch angle of all 100 simulations was $0.127^{\circ}$ for LPILE and $0.149^{\circ}$ for FEM. For the soft soil case, the maximum was $0.168^{\circ}$ for LPILE and $0.174^{\circ}$ for FEM.

Note that these results were based on only 100 ten-minute simulations, which represented the operation of less than a day. The maximum pitch angle will increase further if more simulations are conducted. Considering the 20-year service life of wind turbines, the results and expected increase in the angle indicated that the turbine 
operation of some days may experience serviceability issues because of the pitch angle. To quantify the number of days affected, much more simulations or advanced probabilistic approaches are necessary.

\subsection{Natural frequency}

Another important factor in wind turbine design is natural frequency. The natural frequency of the tower should be different from the frequency of blade rotation to avoid resonance. The 1P frequency is the once per revolution frequency of the blade, and the 3P frequency is the three per revolution frequency of the blade. Most modern turbines have their first natural frequency (of the first side-to-side and fore-aft modes) located between $1 \mathrm{P}$ and $3 \mathrm{P}$. The rotor speed of the wind turbine studied in this paper was 12.1 rpm, and the corresponding $1 \mathrm{P}$ and $3 \mathrm{P}$ frequencies were 0.202 and $0.606 \mathrm{~Hz}$, respectively. Therefore, the first natural frequency should be between these two numbers. The second natural frequency should also be different from the 3P frequency.

Table 5 summarizes the natural frequencies obtained for various soil models. For stiff soil, fixed base assumption overestimated the first mode's natural frequency by $13 \%$ for both the side-to-side mode and fore-aft mode. For soft soil, fixed base assumption over-estimated the first mode's natural frequency by $13 \%$ for the side-toside mode and by $16 \%$ for the fore-aft mode. The difference between the LPILE approach and FEM approach was insignificant (at most 3\% difference). A similar trend was observed for the second mode's frequencies.

\section{Summary and future work}

Offshore wind turbines may experience large base moment of the tower and tilt at the pile head because of wind and wave loading. In order to determine a good modeling approach for the monopile foundation, we compared three different modeling 
approaches. We considered two different soil profiles in this study. Time-domain simulations were conducted using the FAST code. We customized the code to consider the foundation effect. For the 5-MW offshore wind turbine (Jonkman et al. 2009) with rotor diameter of $126 \mathrm{~m}$, tower height of $87.6 \mathrm{~m}$, and water depth of $20 \mathrm{~m}$, the following conclusions were drawn from this study.

(1) The flexibility of soil should be modeled because the fixed base model provided a non-conservative base moment. The fixed base model was also incapable of estimating the tilt at pile head.

(2) For the base moment, both the LPILE approach (based on the p-y curve) and FEM approach (the Mohr-Coulomb model for sand and the Tresca model for clay) provided comparable answers, with less than $4 \%$ difference between the two approaches.

(3) The tilt at pile head increased over 14\% when we used the FEM approach. Therefore, the FEM approach is recommended if the wind turbine experiences serviceability issues caused by the large tilts.

(4) The flexibility of the foundation should be included when computing the natural frequencies. The fixed base model overestimated the first mode's natural frequency by approximately $15 \%$ for both the side-to-side mode and fore-aft mode. However, the approach for modeling the foundation flexibility (LPILE vs. FEM) did not significantly change the natural frequencies, as long as the flexibility of the foundation was included.

These conclusions were based on the simplified estimation of the stiffness presented in Section 4.2. A more accurate approach, particularly considering loading and unloading, may change some of the findings. The conclusions are valid for the wind 
turbine design studied in this paper. The conclusions should not be applied to a different offshore wind turbine, where the results may differ from those of this paper.

Results of the p-y approach in this paper were based on commonly used p-y curves, which were calibrated with long and slender piles. The p-y curve approach in itself is not a problem; however, in this study, conventional p-y curves were calibrated with the piles that were highly different from piles used in offshore wind turbines. When researchers develop newly calibrated p-y curves for offshore wind turbine piles, FEMbased approaches should be involved.

We employed a simplified way of considering soil stiffness in this research. For more accurate analysis, the difference in loading and unloading behaviour of soil should be modeled for the entire time-history of the FAST simulation. Furthermore, the number of simulations should be increased for other purposes such as prediction of long-term load. Given that the tilt at pile head was quite significant, the possibility that it may cause serviceability issues also deserves further study.

\section{Acknowledgments}

The research reported here is supported in part by the National Science Foundation CMMI Grant 1252736 and Basic Science Research Program through the National Research Foundation of Korea (NRF) funded by the Ministry of Education (NRF-2014R1A1A2055858). Any opinions, findings, and conclusions expressed in this paper are those of the authors and do not necessarily reflect the views of the sponsor.

\section{References}

Abdel-Rahman, K. and Achmus, M. (2005), "Finite element modeling of horizontally loaded monopile foundations for offshore wind energy converters in Germany," Frontiers in Offshore Geotechnics: ISFOG, 391-396. 
Achmus, M. and Abdel-Rahman, K. (2012), "Design of piles for offshore wind energy foundations with respect to horizontal loading," Proceedings of the Twenty-second International Offshore and Polar Engineering Conference, Rhodes, Greece.

Achmus, M., Akdag, C.T., and Thieken, K. (2013), "Load-bearing behavior of Suction Bucket Foundations in Sand," Applied Ocean Research, 43, 157-165.

Agarwal, P., and Manuel, L. (2009), "On the modeling of nonlinear waves for prediction of long-term offshore wind turbine loads," Journal of Offshore Mechanics and Arctic Engineering, 131(4), 041601.

API (2007), Recommended Practice for Planning, Design and Constructing Fixed Offshore Platforms-Working Stress Design, American Petroleum Institute.

Bush, E. (2009), A Comparison of Alternative Foundation Models for Offshore Wind Turbines and Resulting Long-Term Loads, M.S. Thesis, the University of Texas at Austin.

Bush, E. and Manuel, L. (2009), "The influence of foundation modeling assumptions on long-term load prediction for offshore wind turbines," Proceedings of the ASME 28th International Conference on Ocean, Offshore and Arctic Engineering, Honolulu, Hawaii, May 31-June 5.

DNV (2011), DNV-OS-J101 Design of Offshore Wind Turbine Structures, Det Norske Veritas.

Chen J.Y., Matarek, B.A., Carpenter J.F., Gilbert, R.B., Verret, S. and Puskar, F.J. (2009), Analysis of Potential Conservatism in Foundation Design for Offshore Platform Assessment, Final Project Report. Contract M08PC20002 MMS Project Number 612.

Hearn, E. N. (2009), Finite Element Analysis of an Offshore Wind Turbine Generator Monopile Foundation, M.S. Thesis, Tufts University. 
Hearn, E.E., and Edgers, L. (2010), "Finite element analysis of an offshore wind turbine monopile," GeoFlorida 2010: Advanced in Analysis, Modeling \& Design (GPS 199), ASCE, 1857-1865.

Hung, L.C., and Kim, S.R. (2014), "Evaluation of undrained bearing capacities of bucket foundations under combined loads," Marine Georesource \& Geotechnology, 32(1), 76-92.

IEC (2005), IEC 61400-1 Wind Turbines - Part 1: Design Requirements, International Electrotechnical Commission, Geneva, Switzerland.

IEC (2009), IEC 61400-3 Wind Turbines - Part 3: Design Requirements for Offshore Wind Turbines, International Electrotechnical Commission, Geneva, Switzerland.

Isenhower, W.M. and Wang, S.T. (2012), Technical Manual for LPILE, Version 6. Ensoft, Inc., Austin, Texas, USA.

Jeanjean, P. (2009), “Re-assessment of p-y curves for soft clays from centrifuge testting and finite element modeling," Offshore Technology Conference, Texas, USA, OTC20158.

Jonkman, J. (2003), Modeling of the UAE Wind Turbine for Refinement of FAST_AD, National Renewable Energy Laboratory, Technical Report No. NREL/TP-50034755.

Jonkman, B. J. (2009), TurbSim User's Guide, Version 1.50, National Renewable Energy Laboratory, Technical Report No. NREL/TP-500-46198.

Jonkman, J. and Buhl, M. L. (2005), FAST User's Guide, National Renewable Energy Laboratory, Technical Report No. NREL/EL-500-38230.

Jonkman, J., Butterfield, S., Musial, W. and Scott, G. (2009), Definition of a 5-MW Reference Wind Turbine for Offshore System Development, National Renewable Energy Laboratory, Technical Report No. NREL/TP-500-38060. 
Jonkman, J., Butterfield, S., Passon, P., Larsen, T., Camp, T., Nichols, J., Azcona, J. and Martinez, A. (2007), "Offshore code comparison collaboration within IEA wind annex XXIII: phase II results regarding monopile foundation modeling," IEA European Offshore Wind Conference, Berlin, Germany, December 4-6.

Kim, D.J., Choo, Y.W., Kim, J.H., Kim, S.R., Kim, D.S. (2014). Investigation of Monotonic and Cyclic behavior of Tripod Suction Bucket Foundations for Offshore Wind Towers Using Centrifuge Modeling. ASCE J. Geotech. Geoenviron. Eng. 140 (5).

Lesny, K., Paikowsky, S. G. and Gurbuz., A. (2007), "Scale effects in lateral load response of large diameter monopiles," Contemporary Issues in Deep Foundations, ASCE.

Lesny, K. and Wiemann, J. (2006), "Finite-element-modelling of large diameter monopiles for offshore wind energy converters,” GeoCongress 2006.

Malhotra, S. (2011), "Selection, design and construction of offshore wind turbine foundations," Wind Turbines, Dr. Ibrahim Al-Bahadly (Ed.), InTech.

Manwell, J. F., McGowan, J. G. and Rogers, A. L. (2009), Wind Energy Explained, 2nd Edition, Wiley.

Matlock, H. (1970), "Correlation for design of laterally loaded piles in soft clays," Proceedings of Offshore Technology Conference, Houston Texas, 577-594.

Moriarty, P. J. and Hansen, A. C. (2005), AeroDyn Theory Manual, National Renewable Energy Laboratory, Technical Report No. NREL/EL-500-36881.

O’Neill, M.W., and Murchison J.M. (1983), An Evaluation of p-y Relationships in Sands. Technical Report to the American Petrolium Institute (PRAC 82-41-1), University of Houston-University Park, Department of Civil Engineering, Research Report No. GT-DF02-83. 
Passon, P. (2006), Memorandum: Derivation and Description of the Soil-PileInteraction Models, IEA-Annex XXIII Subtask 2, Stuttgart, Germany.

Peng, J.R. (2006). Behaviour of Finned Piles in Sand under Lateral Loading. Ph.D thesis, Newcastle University, UK.

Reese, L. C., Cox, W. R. and Koop, F. D. (1975), "Field testing and analysis of laterally loaded piles in stiff clay," Proc., 7th Offshore Technology Conference, Paper No. OTC 2321, Houston, Texas, 671-690.

Simulia (2010), ABAQUS User's Manual, Dassault Systèmes Simulia Corp.TRB (2011), Structural Integrity of Offshore Wind Turbines. Transportation Research Board, Special Report 305.

Sørensen, S. P., Brødbæk, K. T. and Møller, M. (2009), Evaluation of LoadDisplacement Relationships for Large-Diameter Piles, Long Candidate Project Report, Aalborg University.

TRB (2011), Structural Integrity of Offshore Wind Turbines. Transportation Research Board, Special Report 305, p.57.

Van der Tempel, J. (2006), Design of Support Structures for Offshore Wind Turbines, Ph.D. Dissertation, Delft University of Technology.

Zaaijer, M. B. (2002), "Foundation models for the dynamic response of offshore wind turbines," Marine Renewable Energy Conference, Newcastle, UK, September. 
Table 1

Average statistics of shear force and moment: average of 100 ten-minute simulation results (mean wind speed of $12 \mathrm{~m} / \mathrm{s}$, significant wave height of $5 \mathrm{~m}$ )

\begin{tabular}{|l|l|l|l|l|l|}
\hline \multicolumn{2}{|c|}{} & \multicolumn{2}{l|}{ Shear force $(\mathrm{kN})$} & \multicolumn{2}{l|}{ Bending moment $(\mathrm{kN}-\mathrm{m})$} \\
\cline { 2 - 6 } \multicolumn{2}{|l|}{} & Average & Maximum & Average & Maximum \\
\hline \multicolumn{2}{|l}{ Fixed base } & 556.6 & 2328.0 & 62837 & 104856 \\
\hline \multirow{3}{*}{ Stiff soil } & LPILE & 582.7 & 2491.0 & 65350 & 108863 \\
\cline { 2 - 6 } & FEM & 587.9 & 2474.3 & 65853 & 110438 \\
\hline \multirow{2}{*}{ Soft soil } & LPILE & 586.7 & 2484.1 & 65660 & 112258 \\
\cline { 2 - 6 } & FEM & 587.5 & 2521.0 & 65754 & 112170 \\
\hline
\end{tabular}

Table 2

Average statistics of shear force and moment: average of 100 ten-minute simulation results (mean wind speed of $18 \mathrm{~m} / \mathrm{s}$, significant wave height of $5 \mathrm{~m}$ )

\begin{tabular}{|l|l|l|l|l|l|}
\hline \multicolumn{2}{|c|}{} & \multicolumn{2}{l|}{ Shear force $(\mathrm{kN})$} & \multicolumn{2}{l|}{ Bending moment $(\mathrm{kN}-\mathrm{m})$} \\
\cline { 2 - 6 } \multicolumn{2}{|l|}{} & Average & Maximum & Average & Maximum \\
\hline \multicolumn{2}{|l|}{ Fixed base } & 351.3 & 2148.4 & 40249 & 86081 \\
\hline \multirow{3}{*}{ Stiff soil } & LPILE & 356.9 & 2186.7 & 40658 & 90921 \\
\cline { 2 - 6 } & FEM & 354.0 & 2234.9 & 40390 & 87614 \\
\hline \multirow{3}{*}{ Soft soil } & LPILE & 359.5 & 2313.0 & 40806 & 90002 \\
\cline { 2 - 6 } & FEM & 359.7 & 2192.5 & 40761 & 91567 \\
\hline
\end{tabular}

Table 3

Average statistics of surge and pitch: average of 100 ten-minute simulation results (mean wind speed of $12 \mathrm{~m} / \mathrm{s}$, significant wave height of $5 \mathrm{~m}$ )

\begin{tabular}{|l|l|l|l|l|l|}
\hline \multicolumn{2}{|c|}{} & \multicolumn{2}{|l|}{ Surge $(\mathrm{m})$} & Pitch $(\mathrm{deg})$ \\
\cline { 3 - 6 } \multicolumn{2}{|c|}{} & Average & Maximum & Average & Maximum \\
\hline \multirow{3}{*}{ Stiff soil } & LPILE & 0.0087 & 0.0165 & 0.0590 & 0.1075 \\
\cline { 2 - 6 } & FEM & $0.0114(+31.7 \%)$ & $0.0228(+37.9 \%)$ & $0.0674(+14.1 \%)$ & $0.1243(+15.6 \%)$ \\
\hline \multirow{3}{*}{ Soft soil } & LPILE & 0.0165 & 0.0330 & 0.0756 & 0.1454 \\
\cline { 2 - 6 } & FEM & $0.0166(+0.7 \%)$ & $0.0343(+4.0 \%)$ & $0.0813(+7.5 \%)$ & $0.1545(+6.2 \%)$ \\
\hline
\end{tabular}


Table 4

Average statistics of surge and pitch: average of 100 ten-minute simulation results (mean wind speed of $18 \mathrm{~m} / \mathrm{s}$, significant wave height of $5 \mathrm{~m}$ )

\begin{tabular}{|l|l|l|l|l|l|}
\hline \multicolumn{2}{|c|}{} & \multicolumn{2}{|l|}{ Surge $(\mathrm{m})$} & Pitch $(\mathrm{deg})$ \\
\cline { 3 - 6 } \multicolumn{2}{|c|}{} & Average & Maximum & Average & Maximum \\
\hline \multirow{3}{*}{ Stiff soil } & LPILE & 0.0054 & 0.0135 & 0.0367 & 0.0882 \\
\cline { 2 - 6 } & FEM & $0.0070(+29.7 \%)$ & $0.0183(+36.1 \%)$ & $0.0413(+12.5 \%)$ & $0.0991(+12.4 \%)$ \\
\hline \multirow{3}{*}{ Soft soil } & LPILE & 0.0102 & 0.0267 & 0.0469 & 0.1172 \\
\cline { 2 - 6 } & FEM & $0.0103(+0.5 \%)$ & $0.0283(+5.9 \%)$ & $0.0504(+7.3 \%)$ & $0.1263(+7.8 \%)$ \\
\hline
\end{tabular}

Table 5

Natural frequencies of side-to-side and fore-aft modes

\begin{tabular}{|l|l|l|l|l|l|}
\hline \multicolumn{2}{|l|}{} & 1 st s-s & 1 st f-a & 2 nd s-s & 2nd f-a \\
\hline \multirow{2}{*}{ Fixed base } & 0.274 & 0.276 & 1.589 & 1.867 \\
\hline \multirow{2}{*}{ Stiff soil } & LPILE & 0.243 & 0.245 & 1.377 & 1.536 \\
\cline { 2 - 6 } & FEM & 0.242 & 0.241 & 1.366 & 1.489 \\
\cline { 2 - 6 } & LPILE & 0.242 & 0.237 & 1.369 & 1.431 \\
\cline { 2 - 5 } & FEM & 0.234 & 0.235 & 1.337 & 1.417 \\
\hline
\end{tabular}




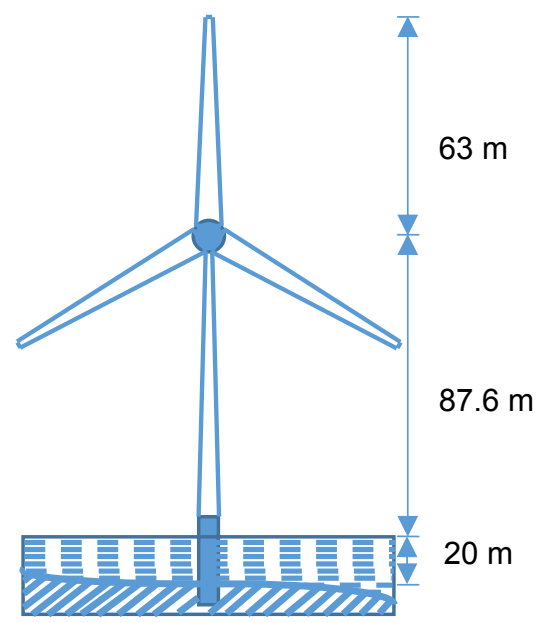

Fig.1. Schematic of the NREL 5-MW offshore wind turbine

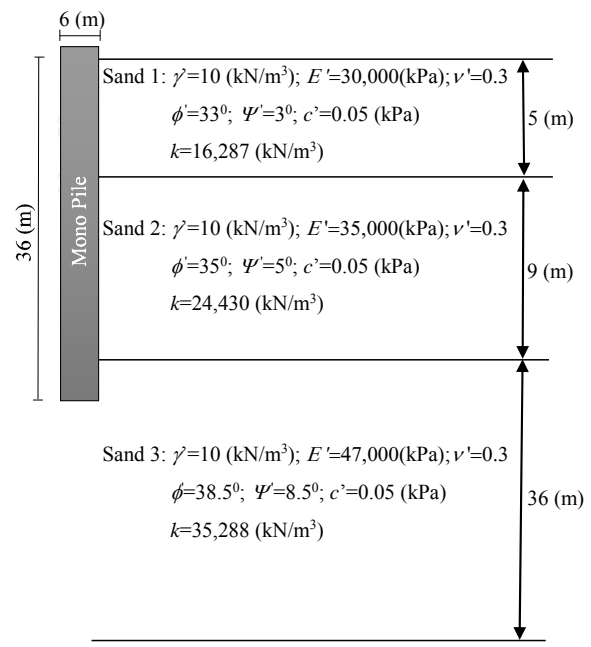

(a) Stiff soil (sand)

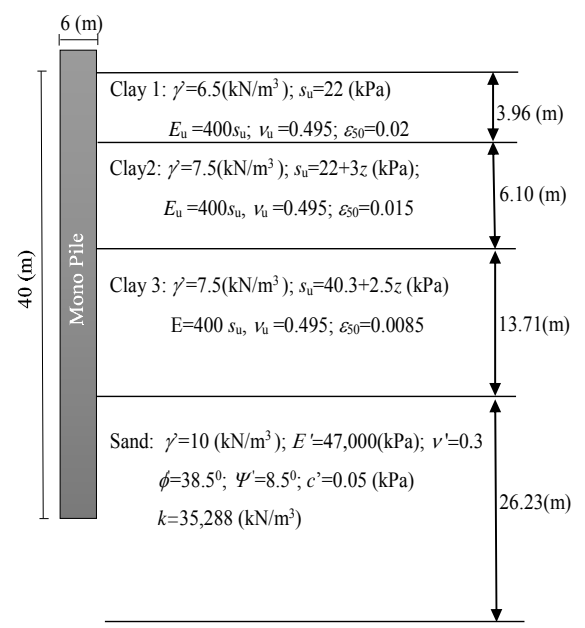

(b) Soft soil (clay and sand)

Fig. 2. Soil profiles and parameters (not to scale) 


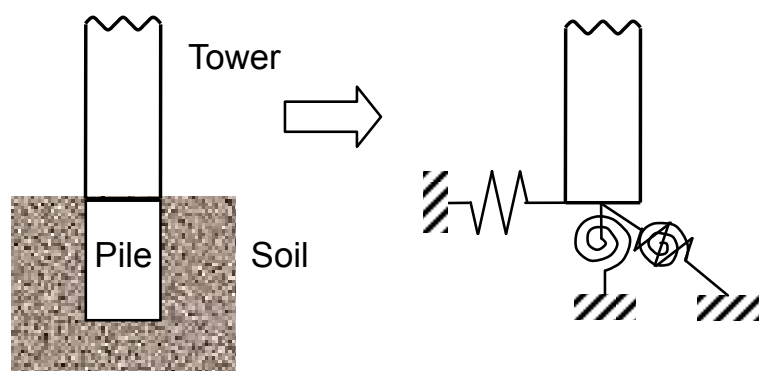

Fig. 3. Coupled spring model to consider the effect of the foundation in wind turbine analysis

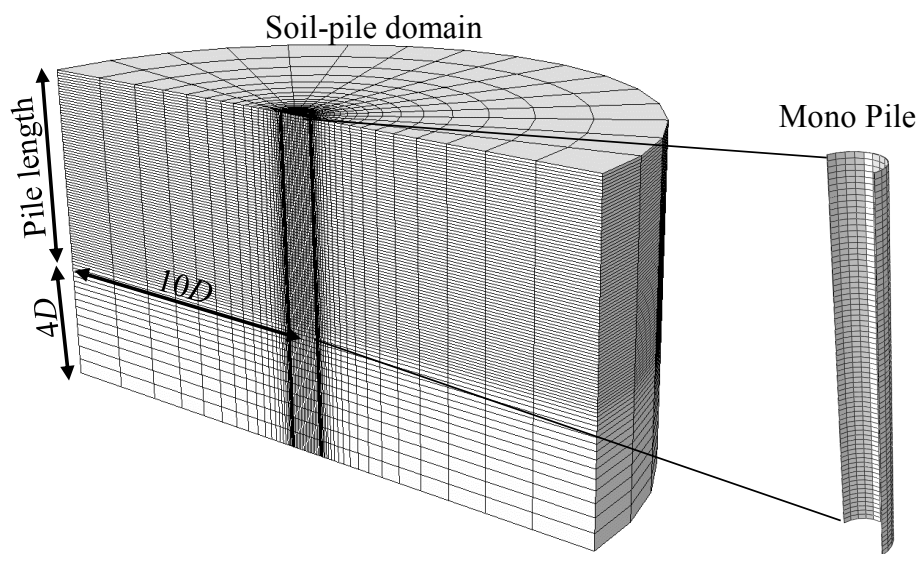

Fig. 4. Typical FE mesh for the soil-pile domain
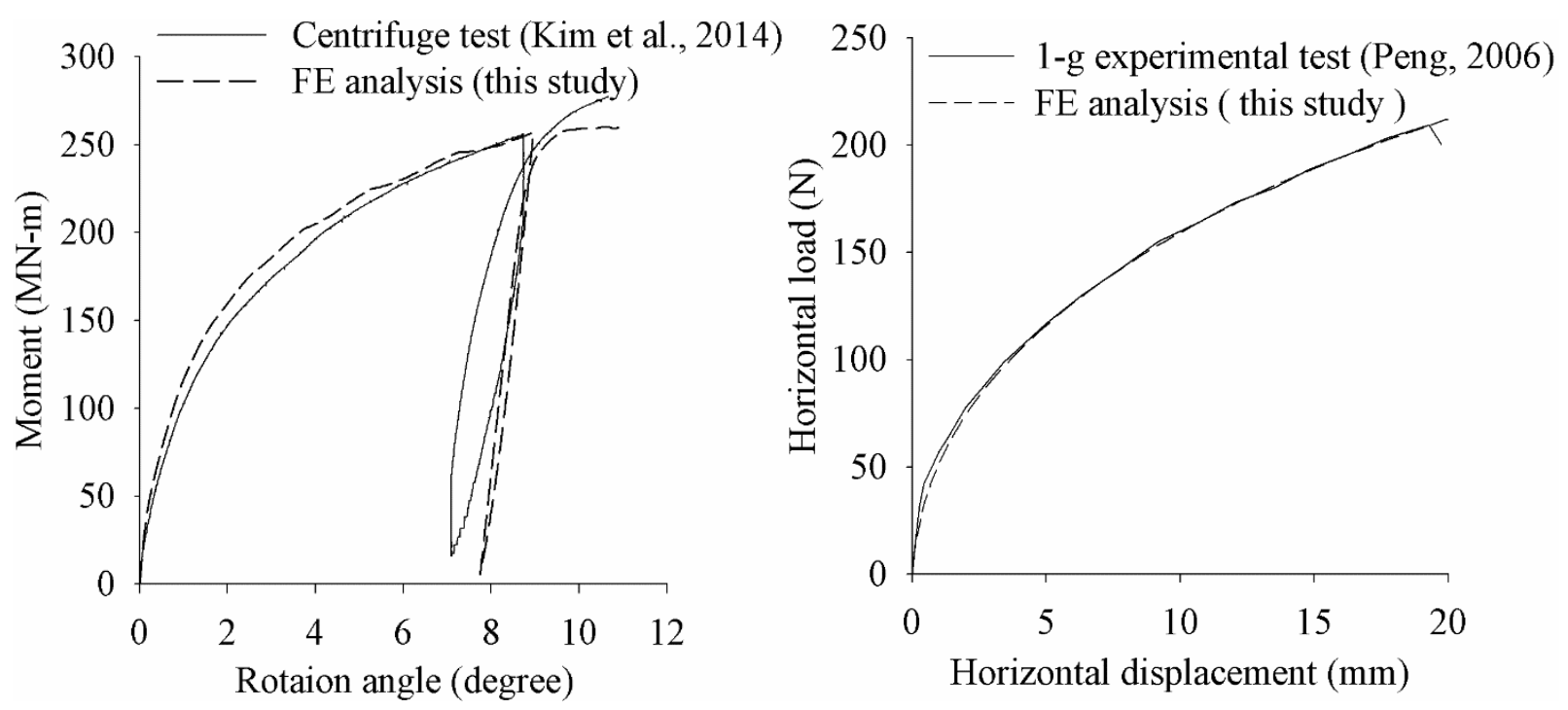

Fig. 5. Comparison of horizontal load-displacement curves between FE analyses and experimental tests 

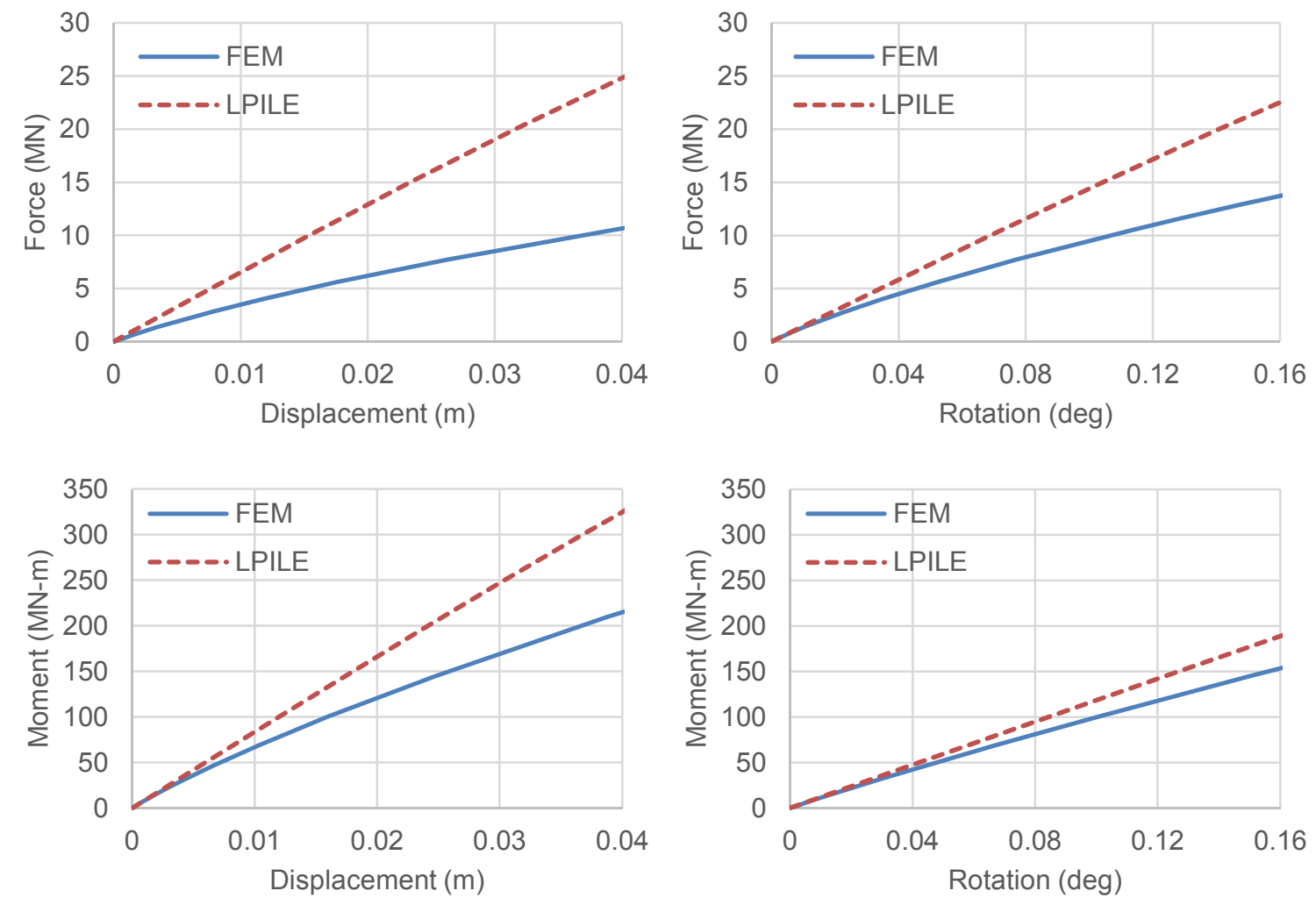

Fig. 6. Responses of the foundation model: stiff soil
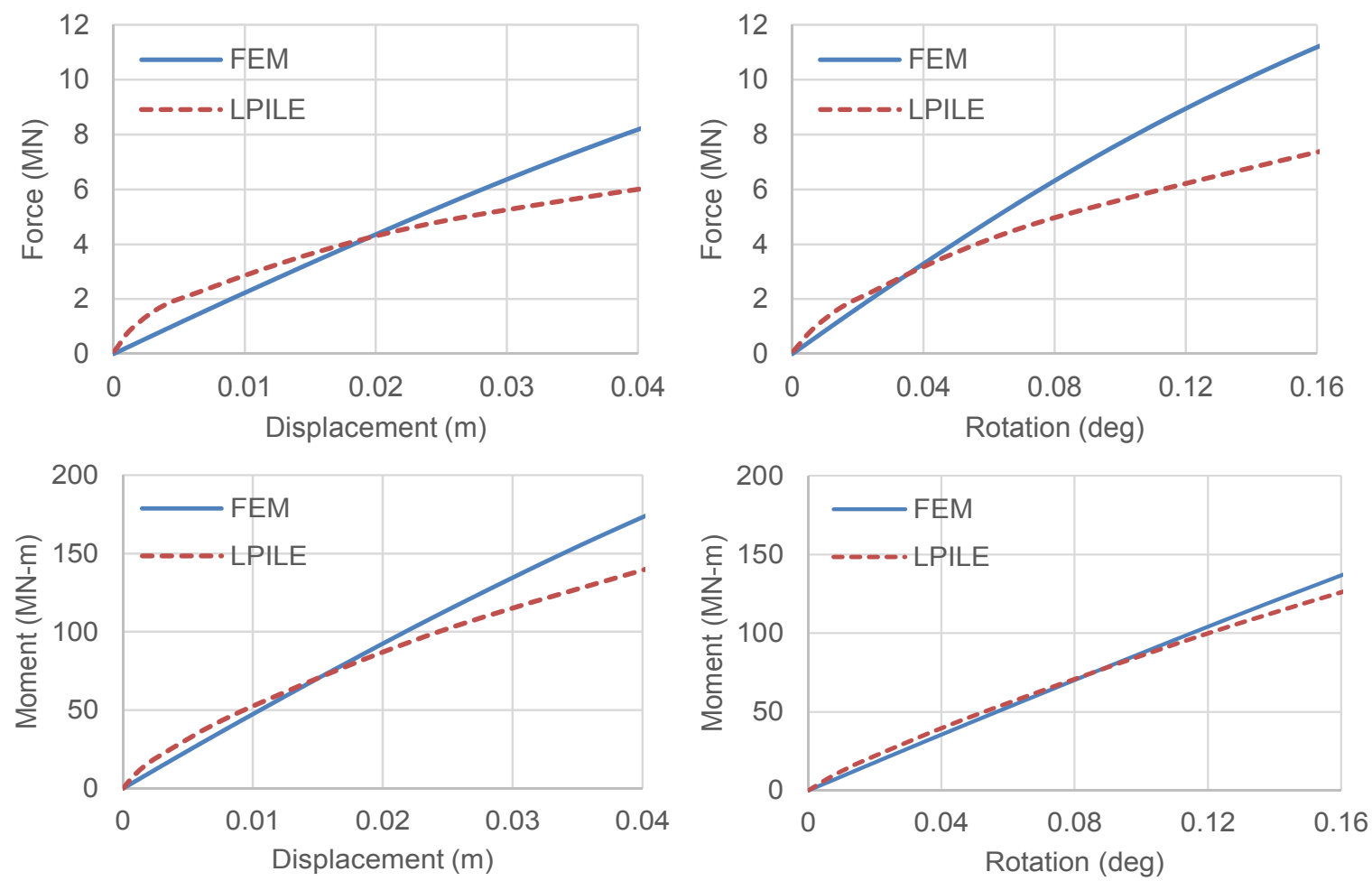

Fig.7. Response of the foundation model: soft soil 


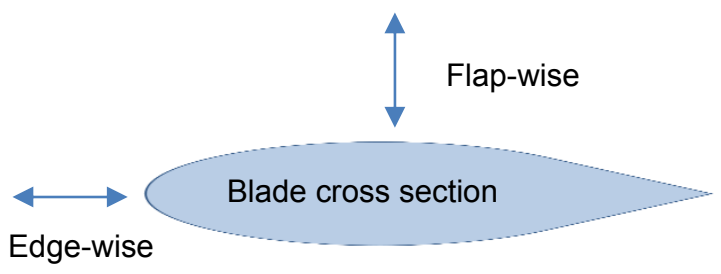

(a) DOFs of the blade

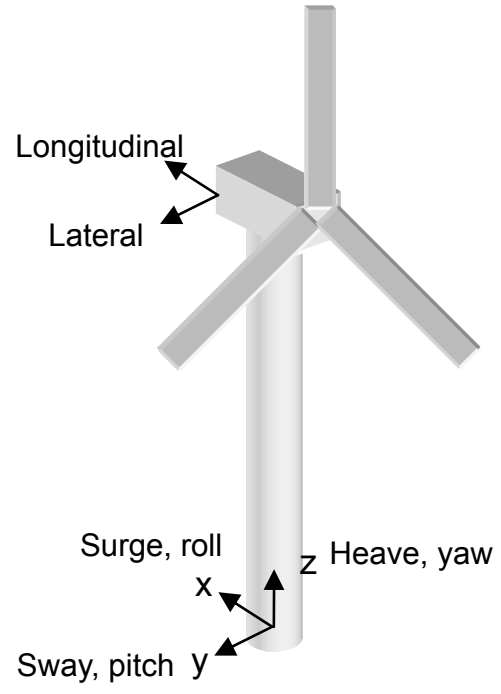

(b) DOFs of the tower and foundation

Fig. 8.DOFsof the blade, tower, and foundation

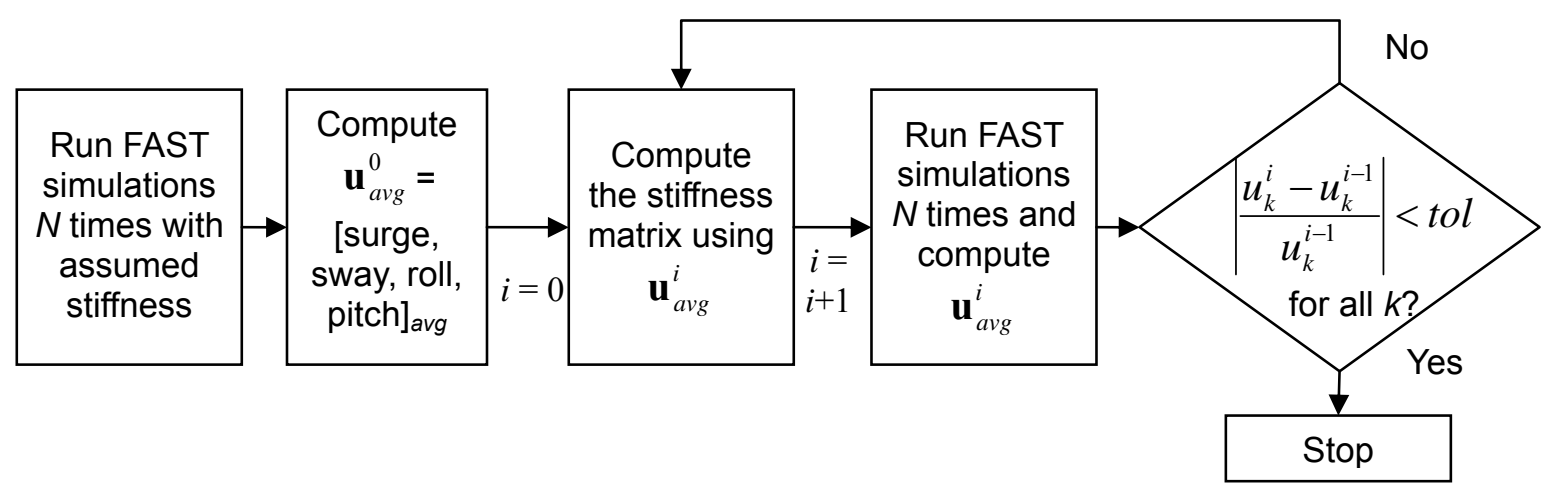

Fig. 9. Flowchart for updating the stiffness matrix of the FAST simulations 

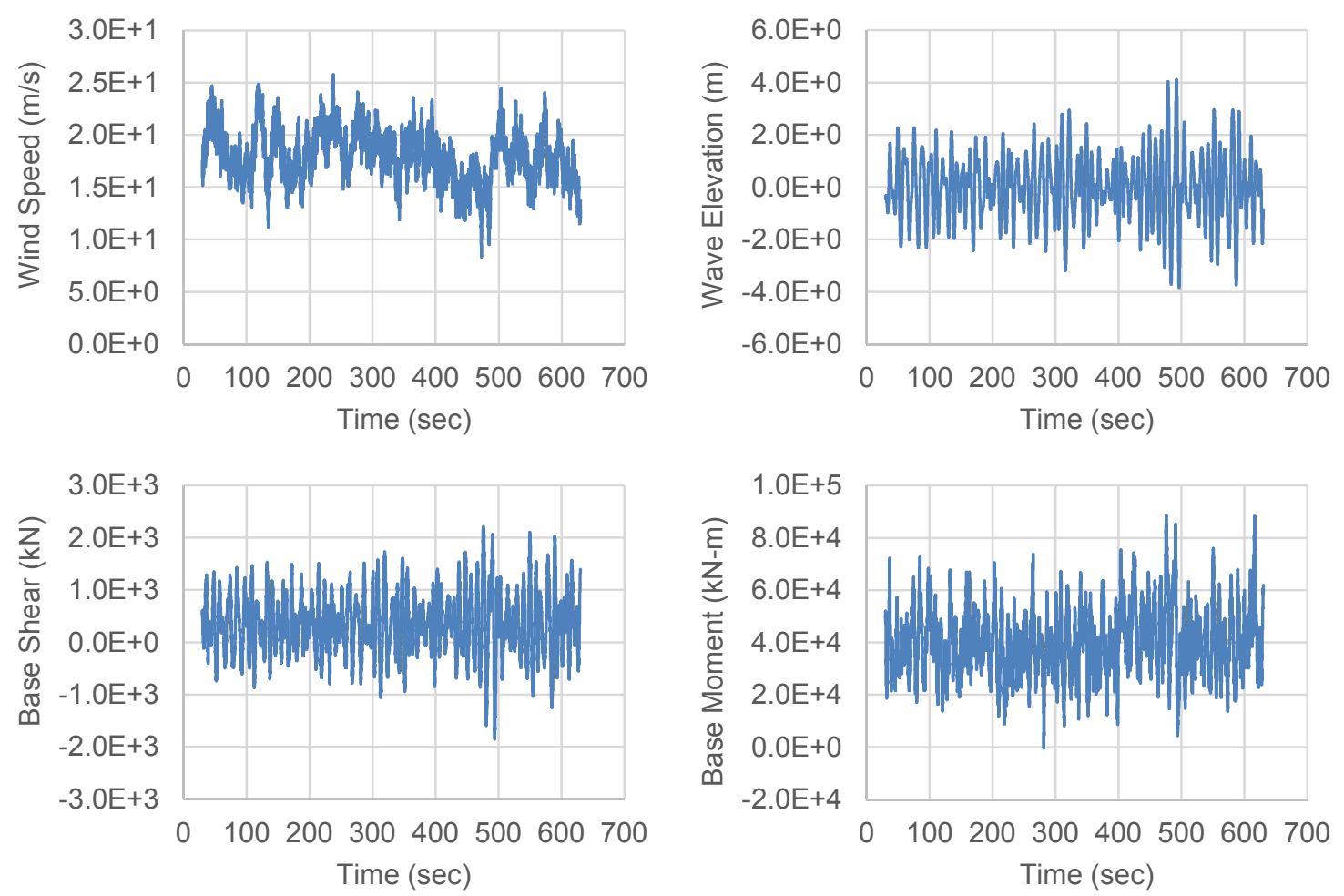

Fig. 10. Example time-domain simulation of a 5-MW offshore turbine (fixed base, mean wind speed of $18 \mathrm{~m} / \mathrm{s}$, significant wave height of $5 \mathrm{~m}$ )

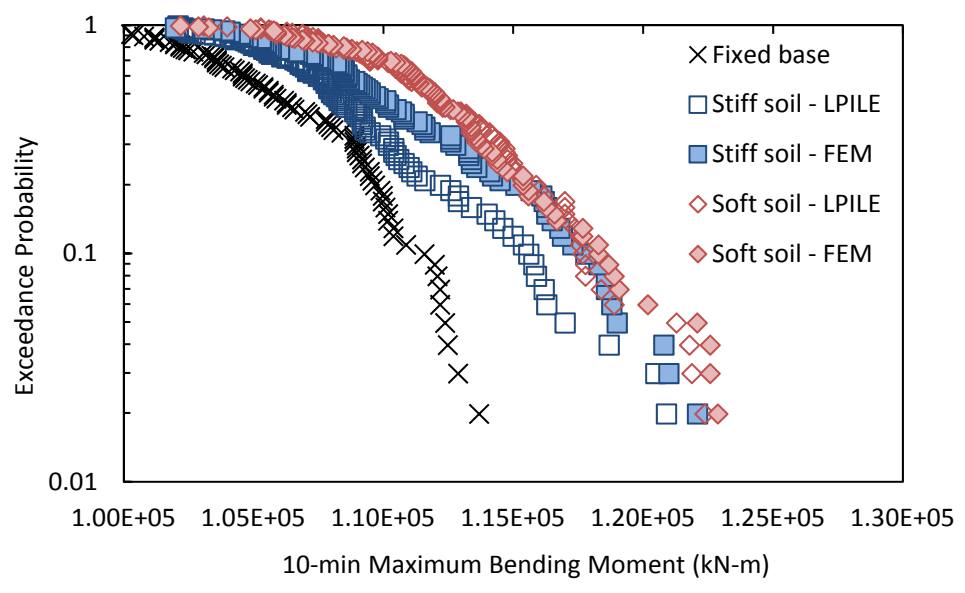

Fig. 11. Exceedance probability of the bending moment (mean wind speed of $12 \mathrm{~m} / \mathrm{s}$, significant wave height of $5 \mathrm{~m}$ ) 


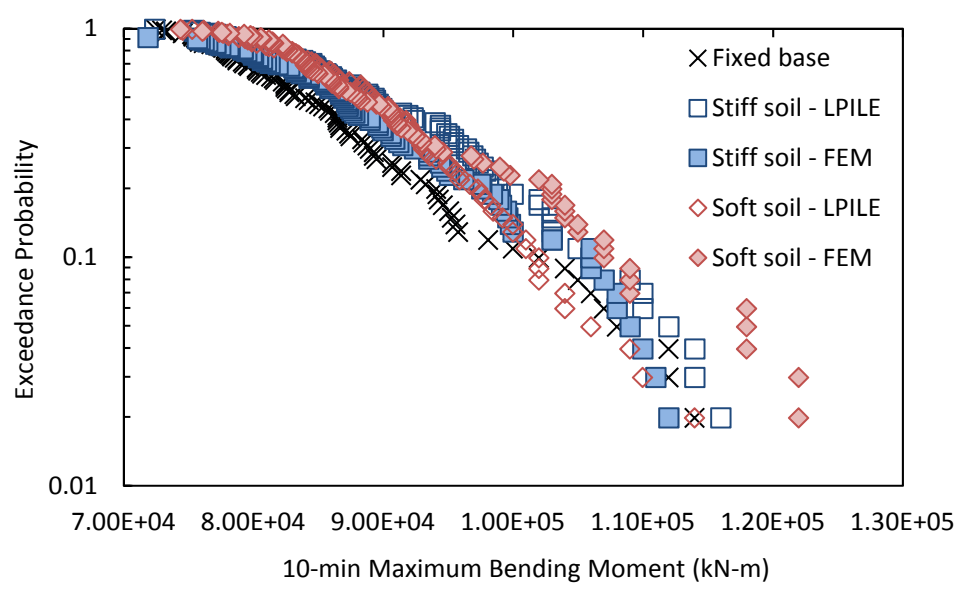

Fig. 12. Exceedance probability of the bending moment (mean wind speed of $18 \mathrm{~m} / \mathrm{s}$, significant wave height of $5 \mathrm{~m}$ )

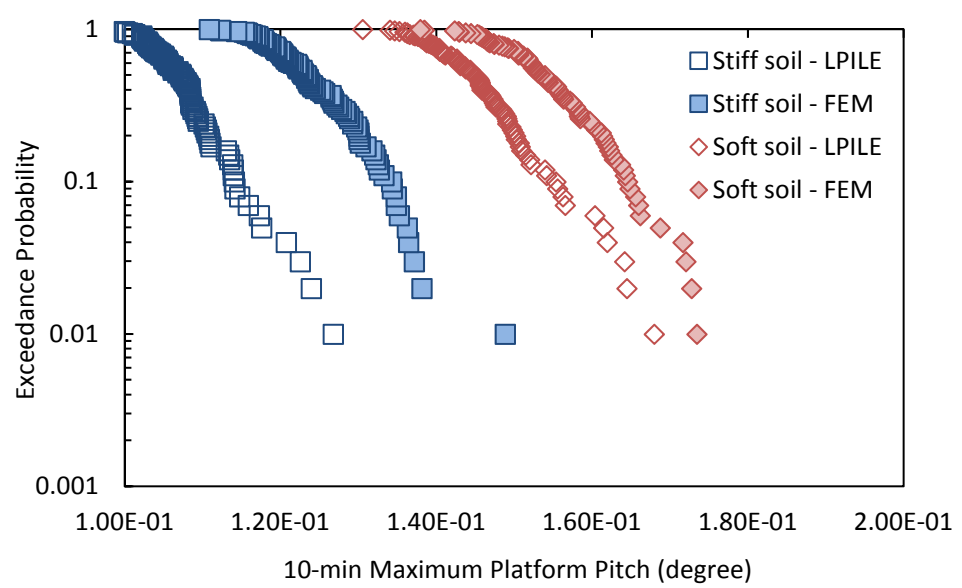

Fig. 13. Exceedance probability of the platform pitch (mean wind speed of $12 \mathrm{~m} / \mathrm{s}$, significant wave height of $5 \mathrm{~m}$ ) 


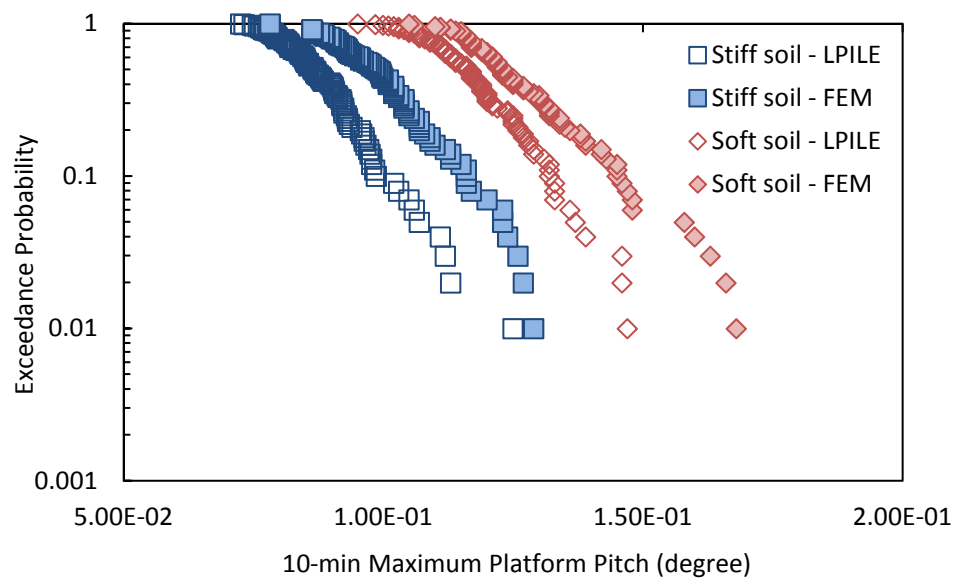

Fig. 14. Exceedance probability of the platform pitch (mean wind speed of $18 \mathrm{~m} / \mathrm{s}$, significant wave height of $5 \mathrm{~m}$ ) 